\title{
Comportamento Ingestivo em Bezerros Holandeses Alimentados com Dietas Contendo Diferentes Níveis de Concentrado ${ }^{1}$
}

\author{
Peter Johann Bürger ${ }^{2}$, José Carlos Pereira ${ }^{3}$, Augusto César de Queiroz ${ }^{3}$, José Fernando Coelho \\ da Silva ${ }^{3}$, Sebastião de Campos Valadares Filho ${ }^{3}$, Paulo Roberto Cecon ${ }^{4}$, Alex Dias Poeta Casali ${ }^{5}$
}

RESUMO - Os efeitos de diferentes níveis de concentrado sobre o comportamento ingestivo foram estudados com cinco bezerros holandeses, inteiros, com idade e peso corporal médios iniciais de 10,8 $\pm 0,8$ meses e 233,4 $\pm 26,1 \mathrm{~kg}$ PV. Os animais foram alojados em baias individuais e alimentados à vontade com dietas contendo 30, 45, 60, 75 e 90\% de concentrado, com base na MS. As rações contendo, aproximadamente, $16 \%$ PB foram formuladas para taxa de ganho de peso de 1,0 kg/dia e compostas por farelo de soja, fubá de milho e feno de capim coast-cross. O delineamento experimental em blocos casualizados com cinco tratamentos, com duração de 20 dias, 12 dias de adaptação e quatro períodos de dois dias, relativos à coleta de dados foi usado. O tempo despendido em alimentação e ruminação diminuiu, e o tempo de ócio aumentou linearmente, em função do aumento dos níveis de concentrado. A eficiência de alimentação em g MS/h registrou comportamento quadrático, estimando-se o valor máximo em 826,81 g FDN/h, para o nível de 60,77\% de concentrado. A elevação no nível de concentrado nas dietas aumentou linearmente a eficiência de ruminação em g MS/h, mas em g FDN/h decresceu linearmente. O número de bolos ruminais e de mastigações merícicas por dia decresceu linearmente. O número e o tempo de mastigação merícica por bolo registraram comportamento quadrático, estimando-se valores máximos de 73,79 mastigações e 66,61 segundos, por bolo ruminal, para os níveis de 48,51 e $54,44 \%$ de concentrado, respectivamente.

Palavras-chave: bezerros holandeses, comportamento ingestivo, concentrado, mastigação merícica, ruminação

\section{Ingestive Behavior in Holstein Calves Fed Diets with Different Concentrate Levels}

\begin{abstract}
The effects of different concentrate levels on the ingestive behavior were studied with five rumen and abomasum fistulated Holstein bull calves, with average initial age of $10.8 \pm 0.8$ months and average $233.4 \pm 26.1 \mathrm{~kg} \mathrm{BW}$. The animals were housed in individual stalls and ad libitum fed diets containing 30,45, 60, 75 and 90\% of concentrate, on DM basis. The diets contained, approximately, $16 \% \mathrm{CP}$ were formulated for body weight gain of $1.0 \mathrm{~kg} / \mathrm{day}$ and constituted of soybean meal corn ground grain in the concentrate and coastcross grass hay as forage. A randomized complete blocks design, with five treatments, throughout 20 days, 12 days of adaptation and four periods of two days, relative to the data collection, was used. The eating and ruminating time decreased, while the idle time linearly increased, as the concentrate levels in the diets increased. The feeding efficiency in $\mathrm{g} D \mathrm{DM} / \mathrm{h}$ showed quadratic behavior, and the maximum estimated value was $826.81 \mathrm{~g} \mathrm{NDF} / \mathrm{h}$, for the level of $60.77 \%$ of concentrate. The rumination efficiency, $\mathrm{g} \mathrm{DM} / \mathrm{h}$, and $\mathrm{g} \mathrm{NDF} / \mathrm{h}$ increased and decreased linearly, respectively, as the concentrate levels in the diets increased. The number of ruminate boli and the ruminating chews per day linearly decreased. The number and the ruminating chews per bolus showed a quadratic behavior, and maximum values of 73.79 ruminating chews and 66.61 seconds, per ruminate boli, were estimated for the levels of 48.51 and $54.44 \%$ of concentrate, respectively.
\end{abstract}

Key Words: Holstein calves, ingestive behavior, concentrate, rumination chews rumination

\section{Introdução}

Segundo Arnold (1985), citado por VAN SOEST (1994), os ruminantes, como outras espécies, procuram ajustar o consumo alimentar às suas necessidades nutricionais, especialmente energia. O comportamento ingestivo de bovinos mantidos a campo caracteriza-se por períodos longos de alimentação, de 4 a 12 horas por dia, entretanto, para animais estabulados, os períodos variam de uma, para alimentos ricos em energia, a seis horas, ou mais, para fontes com baixo teor de energia.

WELCH (1982) afirmou que o aumento no fornecimento de fibra indigestível não incrementa a ruminação a mais de 8 ou 9 h/dia, sendo a eficácia de ruminação importante no controle da utilização de volumosos; assim, um animal que rumina mais volumoso durante esse período de tempo pode consumir mais e ser mais produtivo.

De acordo com THIAGO et al. (1992), a quanti-

\footnotetext{
1 Parte da Tese de Doutorado do primeiro autor.

2 Professor Nível IV do CAV-UDESC, 88520-000 - Lages, SC. E.mail: a2pjb@cav.udesc.br

3 Professor Titular do DZO-UFV. Bolsista do CNPq. E.mail: jearper@mail.ufv.br

4 Professor Adjunto do DPI-UFV.

5 Bolsista Aperfeiçoamento CNPq
} 
dade de alimento consumido por um ruminante, em determinado período de tempo, depende do número de refeições nesse período e da duração e taxa de alimentação de cada refeição. Cada um desses processos é o resultado da interação do metabolismo do animal e das propriedades físicas e químicas da dieta, estimulando receptores da saciedade.

Para dietas volumosas, a mastigação aumenta a degradação ruminal, por elevar a MS e as frações de fibra potencialmente digerível e reduzir o tempo de latência de degradação da fibra. Para dietas de cereais, sabe-se que, quando grãos inteiros não são influenciados na mastigação, a digestão é limitada, e, conseqüentemente, requerem processamento (BEAUCHEMIN, 1992).

O comportamento alimentar tem sido estudado com relação às características dos alimentos, à motilidade do pré-estômago, ao estado de vigília e ao ambiente climático. A diversidade de objetivos e condições experimentais conduziram a várias opções de técnicas de registro dos dados, na forma de observações visuais, registros semi-automáticos e automáticos e parâmetros estudados selecionados para a descrição do comportamento ingestivo, como tempo de alimentação ou ruminação, número de alimentações, períodos de ruminação e eficiência de alimentação e ruminação (DULPHY et al., 1980; FORBES, 1995).

Segundo VAN SOEST (1994), o tempo de ruminação é influenciado pela natureza da dieta e parece ser proporcional ao teor de parede celular dos volumosos. Alimentos concentrados e fenos finamente triturados ou peletizados reduzem o tempo de ruminação, enquanto volumosos com alto teor de parede celular tendem a aumentar o tempo de ruminação. $\mathrm{O}$ aumento do consumo tende a reduzir o tempo de ruminação por grama de alimento, fator provavelmente responsável pelo aumento de tamanho das partículas fecais, quando os consumos são elevados.

O tempo de ruminação é altamente correlacionado $(0,96)$ com o consumo de FDN em bovinos (WELCH e HOOPER, 1988). ALBRIGHT (1993), em experimento com vacas, relatou para três níveis de FDN, nas dietas de 26, 30 e 34\%, resposta quadrática com valores máximos estimados, respectivamente, dos tempos despendidos em ruminação e total de mastigação de 344 e 558; 403 e 651 ; e 414 e $674 \mathrm{~min} /$ dia.

O objetivo deste trabalho foi avaliar os efeitos de diferentes níveis de concentrado na dieta sobre aspectos do comportamento ingestivo em bezerros holandeses.

\section{Material e Métodos}

O local, as condições climáticas do experimento, as instalações e as dietas experimentais, foram descritos por BÜRGER et al. (2000).

Foram utilizados cinco bezerros da raça holandesa, machos, inteiros, puros por cruza, com idade média de $10,8 \pm 0,8$ meses e peso médio inicial de $233,4 \pm 26,1 \mathrm{~kg}$ mantidos em situação de alimentação não-competitiva.

A duração do experimento foi de 20 dias, 12 dias de adaptação às dietas experimentais e à iluminação noturna, e quatro períodos de dois dias cada, relativos à coleta de dados.

No registro do tempo despendido em alimentação, ruminação, ócio e outras atividades, adotou-se a observação visual dos animais a cada cinco minutos, por quatro períodos integrais de 24 horas (JOHNSON e COMBS, 1991).

A média do número de mastigações merícicas por bolo ruminal e a média do tempo despendido de mastigação merícica por bolo ruminal foram obtidas em quatro períodos de oito horas, por quatro dias, nove valores por período, registrando-se três valores distribuídos nos horários das 10 às 12 h, 14 às 16 h e 18 às $20 \mathrm{~h}$, utilizando-se cronômetro digital.

Na observação noturna dos animais, o ambiente foi mantido com iluminação artificial.

Os resultados referentes aos fatores do comportamento ingestivo foram obtidos pelas relações:

$$
\begin{aligned}
\text { EAL } & =\mathrm{CMS} / \mathrm{TAL} \\
\mathrm{ERU} & =\mathrm{CMS} / \mathrm{TRU} \\
\mathrm{ERU} & =\mathrm{CFDN} / \mathrm{TRU} \\
\mathrm{TMT} & =\mathrm{TAL}+\mathrm{TRU} \\
\mathrm{BOL} & =\mathrm{TRU} / \mathrm{MMtb} \\
\mathrm{MM}_{\mathrm{nd}} & =\mathrm{BOLMM}
\end{aligned}
$$

em que EAL (g MS/h) é eficiência de alimentação; CMS (g MS/dia), consumo de MS; TAL (h/dia), tempo de alimentação; ERU (g MS/h; g FDN/h), eficiência de ruminação; TRU (h/dia), tempo de ruminação; TMT (h/dia), tempo de mastigação total; $\mathrm{BOL}$ ( $\mathrm{n}$ \%/dia), número de bolos ruminais; TRU (s/ dia), tempo de ruminação; $\mathrm{MM}_{\mathrm{tb}}$ (s/bolo), tempo de mastigações merícicas por bolo ruminal (POLLI et al., 1996); $\mathrm{MM}_{\mathrm{nd}}$ (n\%/dia), número de mastigações merícicas; $\mathrm{e} \mathrm{MM}_{\mathrm{nb}}(\mathrm{n} \mathrm{o} / \mathrm{bolo})$, número de mastigações merícicas por bolo.

Utilizou-se delineamento experimental em blocos casualizados com cinco tratamentos, correspondendo aos níveis de 30, 45, 60, 75 e $90 \%$ de concentrado e quatro repetições. 


\section{Rev. bras. zootec.}

Os dados experimentais foram analisados empregando-se o programa SAEG - Sistema de Análises Estatísticas e Genéticas, versão 7.1 (UNIVERSIDADE FEDERAL DE VIÇOSA - UFV, 1997).

Os resultados foram interpretados estatisticamente por meio de análises de variância e regressão, adotando-se o nível de 5\% de probabilidade.

Os modelos foram selecionados com base no coeficiente de determinação e na significância dos coeficientes de regressão, adotando-se o nível de 5\% de probabilidade, utilizando-se o teste $\mathrm{F}$.

\section{Resultados e Discussão}

Os resultados relativos aos tempos médios despendidos em alimentação, ruminação, ócio e outras atividades, em horas por dia (h/dia), e respectivas equações de regressão ajustadas e coeficientes de determinação são apresentados na Tabela $1 \mathrm{e}$, em $\%$, na Figura 1.

Os tempos de alimentação e ruminação diminuíram linearmente $(\mathrm{P}<0,01)$, com o tempo de ócio, elevando-se linearmente $(\mathrm{P}<0,05)$; contudo, as outras atividades não foram influenciadas, apresentando valor médio de $0,77 \mathrm{~h} / \mathrm{dia}$, em função dos níveis de concentrado.

TRAXLER et al. (1995), que alimentaram bezerros holandeses com peso corporal médio de $137 \mathrm{~kg}$, com $85 \%$ de grãos de milho inteiros mais volumoso peletizado e $90 \%$ de grãos de milho inteiro ou moído mais silagem constituída de gramíneas e leguminosas, durante a fase de crescimento, relataram, respectivamente, intervalos para o tempo de alimentação de 2,76 a 2,21 h/dia e de ruminação, 5,66 a 4,36 h/dia, relativamente próximos dos valores encontrados, quando as dietas continham 75 e $90 \%$ de concentrado (Tabela 1).

DULPHY et al. (1980) relataram que, aumentando a proporção de concentrado nas dietas, o tempo despendido em ruminação decresceu. Este fato pode ser observado na Tabela 1, em que os dados apresentam decréscimo linear, em função do aumento no nível de concentrado.

MOORE et al. (1990), que trabalharam com novilhos alimentados com palha de trigo suplementada com $65 \%$ de concentrado, registrando o comportamento ingestivo com observação visual a cada 5 minutos por 24 horas, relataram tempo de ruminação de 5,42 h/dia, intermediário aos valores de 6,83 e 4,23 h/dia para o intervalo dos níveis de 60 e $90 \%$ de concentrado obtidos neste trabalho.

KENNEDY et al. (1992), trabalhando com novi-

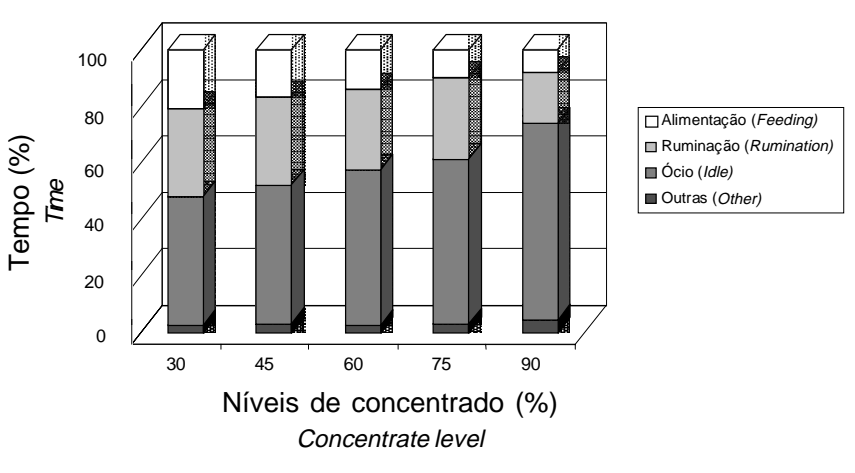

Figura 1 - Padrão do comportamento alimentar dos tempos diários despendidos em alimentação, ruminação, ócio e outras atividades, em função dos níveis de concentrado (\%) das dietas.

Figura 1 - Ingestive behavior pattern of the daily spending times with feeding, rumination, idle and other activities, according to the concentrate levels of the diets.

lhas mestiças alimentadas com palha de arroz (Oriza sativa $\mathrm{cv}$. Bluebonnet L.) suplementada com concentrado à base de farelo de girassol, relataram tempo de ócio de 10,27 h/dia, valor similar a 10,92 h/ dia referente ao nível de $30 \%$ de concentrado.

Os resultados concernentes ao consumo de MS, em g/dia e \%PV, ao consumo de FDN, em g/dia, à eficiência de alimentação, em g MS/h e g FDN/h, à eficiência de ruminação da dieta completa, em $\mathrm{g} M S / \mathrm{h}$ e g FDN/h, e à eficiência de ruminação da fração de volumoso, em $\mathrm{g} \mathrm{MS} / \mathrm{h}$, e respectivas equações de regressão ajustadas e coeficientes de determinação são apresentados na Tabela 2 .

$\mathrm{O}$ consumo em g MS/dia e \%PV apresentou comportamento quadrático, com estimativas dos valores máximos de 6.193,37 g/dia e 2,49\% PV, para os níveis de concentrado de 64,16 e $43,26 \%$, respectivamente, ao passo que o consumo de FDN, em g/dia foi reduzido linearmente $(\mathrm{P}<0,01)$, com os níveis de concentrado.

A eficiência de alimentação, quando expressa em $\mathrm{g} \mathrm{MS} / \mathrm{h}$, registrou crescimento linear $(\mathrm{P}<0,01)$, porém, expressa em g FDN/h, apresentou comportamento quadrático, com estimativa de valor máximo de $826,81 \mathrm{~g} \mathrm{FDN} / \mathrm{h}$, para o nível de inclusão de concentrado de $60,77 \%$.

A eficiência de ruminação da dieta completa, expressa em g MS/h, aumentou linearmente $(\mathrm{P}<0,01)$; contudo, quando determinada apenas sobre a fração de volumoso da dieta, decresceu linearmente $(\mathrm{P}<0,05)$. Quando expressa em g FDN/h, também diminuiu linearmente $(\mathrm{P}<0,01)$ (Tabela 2). 
BÜRGER et al.

Tabela 1 - Médias do tempo despendido em alimentação, ruminação, ócio e outras atividades em função dos níveis de concentrado das dietas

Table 1 - Average spending time with feeding, rumination, idle and other activities, according to concentrate levels in the diets

Atividades (h/dia)

Activities (h/day)

Alimentação(Feeding)

Ruminação (Rumination)

Ócio(Idle) Níveis de concentrado (\%)

\begin{tabular}{cccccc}
\multicolumn{5}{c}{ Níveis de concentrado (\%) } & ER \\
\cline { 1 - 4 } Concentrate levels & RE \\
\hline 40 & 45 & 60 & 75 & 90 & \\
7,92 & 3,96 & 3,29 & 2,31 & 1,92 & 1 \\
10,92 & 7,54 & 6,83 & 6,94 & 4,23 & 2 \\
0,64 & 11,69 & 13,25 & 14,02 & 16,79 & 3 \\
\cline { 1 - 4 } & 0,81 & 0,63 & 0,73 & 1,06 & $\hat{\mathrm{Y}}=0,77$ \\
\hline
\end{tabular}

1. $\hat{\mathrm{Y}}=6,3380-0,05099^{\star *} \mathrm{C}, \mathrm{r}^{2}=0,99 ; 2 . \hat{\mathrm{Y}}=9,4875-0,04792^{\star *} \mathrm{C}, \mathrm{r}^{2}=0,69 ; 3 . \hat{\mathrm{Y}}=7,70+0,09389^{\star *} \mathrm{C}$, $r^{2}=0,94 ;{ }^{* *}(\mathrm{P}<0,01)$ pelo teste $\mathrm{F}$ (by F test); e C = Nível de concentrado nas dietas experimentais (Concentrate level in the experimental diets).

Tabela 2 - Médias dos consumos de MS (CMS e CMS PV $)$ e FDN (CFDN), eficiência de alimentação de $M S(E A L)$ e de FDN ( $E A L_{F D N}$ ), eficiência de ruminação da dieta total (ERU), eficiência de ruminação da fração de volumoso $\left(E R U_{V}\right)$ e eficiência de ruminação de FDN $\left(E R U_{\mathrm{FDN}}\right)$, em função dos níveis de concentrado das dietas

Table 2 - Means of the intakes of DM (DMI) and (DMI $\mathrm{LW}_{\text {) }}$ and NDF (NDFI), feeding efficiency of DM (FEF) and NDF (FEF $\left.F_{N D F}\right)$, rumination efficiency of the total diet (RUE) and rumination of the bulky fraction $\left(R U E_{r}\right)$ according to the concentrate levels in the diets

\begin{tabular}{|c|c|c|c|c|c|c|}
\hline \multirow[t]{2}{*}{ Item } & \multicolumn{5}{|c|}{$\begin{array}{c}\text { Níveis de concentrado }(\%) \\
\text { Concentrate levels }\end{array}$} & \multirow[t]{2}{*}{$\begin{array}{l}\mathrm{ER} \\
R E\end{array}$} \\
\hline & 30 & 45 & 60 & 75 & 90 & \\
\hline CMS (DMI) (g/dia) & $5.071,27$ & $5.950,46$ & $5.605,32$ & $6.716,94$ & $5.306,59$ & 1 \\
\hline $\mathrm{CMS}_{\mathrm{PV}}\left(D M I_{L W}\right)(\% \mathrm{PV})$ & 2,45 & 2,52 & 2,44 & 2,36 & 2,18 & 2 \\
\hline CFDN (g/dia) & $3.089,77$ & $3.046,96$ & $2.266,79$ & $2.078,56$ & $1.086,05$ & 3 \\
\hline EAL (g MS/h) & $1.034,91$ & $1.528,19$ & $1.759,51$ & $2.988,36$ & $2.860,05$ & 4 \\
\hline $\mathrm{EAL}_{\mathrm{FDN}}(F E F)(\mathrm{g} \mathrm{FDN} / \mathrm{h})$ & 630,54 & 782,52 & 711,55 & 924,75 & 585,33 & 5 \\
\hline $\operatorname{ERU}(R U E)(\mathrm{g} \mathrm{MS} / \mathrm{h})$ & 700,65 & 788,88 & 934,36 & 988,81 & $1.456,80$ & 6 \\
\hline $\mathrm{ERUV}^{\mathrm{V}}\left(R U E_{r}\right)(\mathrm{g} \mathrm{MS} / \mathrm{h})$ & 490,45 & 433,88 & 373,74 & 247,20 & 145,68 & 7 \\
\hline $\mathrm{ERU}_{\mathrm{FDN}}\left(R U E_{N D F}\right)(\mathrm{g} \mathrm{FDN} / \mathrm{h})$ & 426,88 & 403,95 & 377,85 & 305,99 & 298,15 & 8 \\
\hline
\end{tabular}

1. $\hat{Y}=2112,95+127,193^{\star} C-0,9912^{\star *} C^{2}, R^{2}=0,51 ; 2 . \hat{Y}=2,1878+0,01381^{\star *} C-0,0001596^{*} C^{2}, r^{2}=0,98 ; 3$. $\hat{\mathrm{Y}}=4303,97-33,1723^{* \star} \mathrm{C}, \mathrm{r}^{2}=0,92 ; 4 . \hat{\mathrm{Y}}=-9,9762+34,0697^{\star *} \mathrm{C}, \mathrm{r}^{2}=0,89 ; 5 . \hat{\mathrm{Y}}=7,5919+26,9594^{\mathrm{ns}} \mathrm{C}-0,2218^{*} \mathrm{C}^{2}$, $R^{2}=0,49 ; 6 . \hat{Y}=289,0062+11,4149^{\star *} \mathrm{C}, r^{2}=0,85 ; 7 . \hat{Y}=688,6840-5,8415^{\star *} C, r^{2}=0,97 ; 8 . \hat{Y}=504,738$ $-2,3695^{*} C, r^{2}=0,94 ;{ }^{*} e^{* *}(P<0,05)$ e $(P<0,01)$ pelo teste $F$ (by $F$ test); ns $(P>0,05)$ pelo teste $F$ (by $F$ test); e $\mathrm{C}=$ Nível de concentrado nas dietas (Concentrate level in the diets).

De acordo com DULPHY et al. (1980), elevando-se a inclusão de concentrado da dieta, aumenta a eficiência de ruminação; calculada da fração do volumoso, decresce com o aumento do concentrado, sendo provavelmente ainda menor que a estimada, devido ao declínio progressivo da atividade celulolítica dos microrganismos ruminais e ao fato de que parte do concentrado é regurgitado no bolo durante a ruminação (Tabela 2).

As médias do tempo de mastigação total, em horas por dia, do número de bolos ruminais e do número de mastigações merícicas, em $\mathrm{n}$ \%/dia, do número de mastigações merícicas por bolo, em $\mathrm{n}$ ㅇ bolo, e do tempo de mastigação merícica por bolo, em s/bolo, as equações de regressão ajustadas e os coeficientes de determinação, em função dos níveis de concentrado, são apresentados na Tabela 3.

O tempo de mastigação total e o número de bolos ruminais e de mastigações merícicas por dia diminuíram linearmente $(\mathrm{P}<0,01, \mathrm{P}<0,05$ e $\mathrm{P}<0,01$, respectivamente), enquanto o número e o tempo de mastigações merícicas por bolo ruminal apresentaram comportamento quadrático, com estimativas de valores máximos de 73,79 mastigações por bolo e 66,61 segundos por bolo, correspondendo aos níveis de 48,51 e $54,44 \%$ de concentrado.

Segundo DULPHY et al. (1980), quando decrescem os constituintes da parede celular da dieta, aumentando o teor de amido, decresce o tempo total de mastigação, o que pode ser observado com a redução linear verificada na Tabela 3 .

BEAUCHEMIN (1991), que trabalhou com va- 
cas holandesas alimentadas com feno de alfafa, ajustando as dietas para três concentrações de FDN $(31,34$, e $37 \%)$, suplementado com concentrado à base de cevada, relatou tempos de mastigação total, respectivamente, de 12,$8 ; 12,75$; e $13,13 \mathrm{~h} / \mathrm{dia}$, valores relativamente próximos à média de $12,44 \mathrm{~h} / \mathrm{dia}$ observada na Tabela 3.

ALLEN (1997), em revisão da literatura, relatou os resultados de 132 tratamentos, médias de 32 experimentos para o tempo de mastigação total, mencionando o valor de $11,13 \mathrm{~h} /$ dia, intermediário às médias de 11,50 e 10,12 h/dia, correspondentes aos níveis de 45 e $60 \%$ de concentrado.

MacLEOD et al. (1994) verificaram, em novilhas holandesas alimentadas duas vezes ao dia, com silagem de alfafa e concentrado à base de farelo de soja e fubá de milho, tempo de mastigação total de $10,05 \mathrm{~h} /$ dia similar ao valor de $10,12 \mathrm{~h} /$ dia observado na Tabela 3, para 60\% de concentrado na dieta.

BEAUCHEMIN e IWAASA (1993), que utilizaram novilhas da raça Hereford alimentadas com capim-dos-pomares (Dactylis glomerata L.) em dois estádios de maturidade, relataram número de bolos ruminais, de 397 e 350 ao dia, próximo às médias de 414,60 e 356,80 obtidas para os níveis de 45 e $60 \%$ de concentrado, e 58,1 mastigações merícicas por bolo, próximo do valor de 58,89 mastigações, quando a dieta continha $75 \%$ de concentrado (Tabela 3). Em revisão de literatura, HAFEZ e BOUISSON (1975) encontraram o número médio de 360 bolos ruminais nas 24 horas, similar ao valor de 356,80 bolos/dia registrado na Tabela 3, para $60 \%$ de concentrado na dieta. BAE et al. (1981) mencionaram a média de 462 bolos ruminais, intermediária aos valores de 482,60 e 414,81 bolos ao dia, correspondentes aos níveis de 30 e $45 \%$ de concentrado.

Os números de mastigações merícicas diárias de $31,49 \times 10^{3}$ e $31,89 \times 10^{3}$ observados para os níveis de 45 e $30 \%$ de concentrado (Tabela 3 ) foram similares aos relatados por BOSCH et al. (1992), que trabalharam com vacas leiteiras alimentadas com duas silagens de gramíneas (44,6 e 54,7\% FDN), encontrando, respectivamente, $31,24 \times 10^{3}$ e $31,77 \times 10^{3}$ mastigações merícicas diária.

As médias do número de refeições diárias (ou visitas ao comedouro) e o tempo despendido por refeição, em minutos, são apresentados na Tabela 4. O número de refeições diárias não foi influenciado pelos níveis de concentrado, observando-se o valor médio de 14,80, enquanto, para o tempo despendido por refeição, ocorreu efeito linear decrescente $(\mathrm{P}<0,01)$, em função dos níveis de concentrado.

JOHNSON e COMBS (1991), que testaram vacas holandesas em lactação, alimentadas com silagem de alfafa e silagem de milho e suplementadas com farelo de soja, registraram o tempo despendido por refeição de 14,7 minutos, próximo da média de 13,33 minutos obtida para os níveis de 45 e $60 \%$ de concentrado, e o número de 18 refeições ao dia foi similar ao valor médio de 18,25 , observado nos animais que receberam rações com $45 \%$ de concentrado (Tabela 4 ).

O valor médio de 14,80 de refeições ao dia, observado na Tabela 4 , foi próximo da média de 15,33 visitas aos comedouros relatadas por LIVSHIN et al. (1995), que trabalharam com 50 vacas holandesas suplementadas com concentrado, utilizando sistema computadorizado de alimentação, para atender as exigências energéticas individuais da produção de leite.

A distribuição média da freqüência do número de

Tabela 3 - Médias do tempo de mastigação total (TMT), do número de bolos ruminais (NBR), do número de mastigações merícicas $\left(\mathrm{MM}_{\mathrm{nd}}\right)$, das mastigações merícicas por bolo $\left(\mathrm{MM}_{\mathrm{nb}}\right)$ e do tempo de mastigações merícicas por bolo $\left(\mathrm{MM}_{\mathrm{tb}}\right)$, em função dos níveis de concentrado das dietas

Table 3 - Means of the total chewing time (TCT), number of rumination boli (NRB), number of rumination chews $\left(R C_{n d}\right)$, number of rumination chews per bolus $\left(R C_{n b}\right)$ and rumination chews time per bolus $\left(R C_{t b}\right)$, according to the concentrate levels in the diets

\begin{tabular}{|c|c|c|c|c|c|c|}
\hline \multirow[t]{2}{*}{ Item } & \multicolumn{5}{|c|}{$\begin{array}{l}\text { Níveis de concentrado (\%) } \\
\text { Concentrate levels }\end{array}$} & \multirow[t]{2}{*}{$\begin{array}{l}\mathrm{ER} \\
R E\end{array}$} \\
\hline & 30 & 45 & 60 & 75 & 90 & \\
\hline TMT (TCT) (h/dia) & 12,44 & 11,50 & 10,12 & 9,20 & 6,14 & 1 \\
\hline $\operatorname{NBR}(N R B)(\mathrm{n} \div / \mathrm{dia})$ & 482,60 & 414,81 & 356,80 & 446,66 & 308,42 & 2 \\
\hline $\operatorname{MMnd}\left(R C_{n d}\right)(103)(\mathrm{n}-/ \mathrm{dia})$ & 31,89 & 31,49 & 26,58 & 26,14 & 15,67 & 3 \\
\hline $\operatorname{MMnb}\left(R C_{n b}\right)(\mathrm{n} \underline{\mathrm{o}} / \mathrm{bolo})$ & 67,36 & 76,14 & 73,72 & 58,89 & 51,47 & 4 \\
\hline $\operatorname{MMtb}\left(R C_{t b}\right)(\mathrm{s} / \mathrm{bolo})$ & 57,20 & 65,78 & 69,35 & 55,78 & 50,11 & 5 \\
\hline
\end{tabular}

1. $\hat{\mathrm{Y}}=15,8333-0,09917^{\star *} \mathrm{C}, \mathrm{r}^{2}=0,94 ; 2 . \hat{\mathrm{Y}}=528,462-2,1100^{*} \mathrm{C}, \mathrm{r}^{2}=0,51 ; 3 . \quad \hat{\mathrm{Y}}=41475,6220-252,0177^{\star *} \mathrm{C}$, $r^{2}=0,83 ; 4 . \hat{Y}=40,3280+1,3797^{* \star} C-0,01422^{* \star} C^{2}, R^{2}=0,85 ; 5 . \hat{Y}=23,6740+1,5776{ }^{* *} C-0,0144905^{* *} C^{2}$, $R^{2}=0,85 ;{ }^{*} e^{* *}(P<0,05)$ e $(P<0,01)$ pelo teste $F$ (by $F$ test); e $C=$ Nível de concentrado nas dietas (Concentrate level in the diets). 
BÜRGER et al.

refeições por dia, em relação ao tempo despendido por refeição em minutos, pode ser observada na Tabela 5 e, em \%, na Figura 2. Observa-se na Tabela
5 que os intervalos de 5 a 14,15 a 24 e 25 a mais de 55 minutos concentraram, respectivamente, 63,51; 22,98; e 13,51\% do número de refeições por dia.

Tabela 4 - Médias do número de refeições diárias e do tempo despendido por refeição, em função dos níveis de concentrado das dietas

Table 4 - Means of the daily meals number and of the spending time per meal, according to the concentrate levels in the diets

\begin{tabular}{|c|c|c|c|c|c|c|}
\hline \multirow[t]{2}{*}{ Item } & \multicolumn{5}{|c|}{$\begin{array}{c}\text { Níveis de concentrado (\%) } \\
\text { Concentrate levels }\end{array}$} & \multirow[t]{2}{*}{$\begin{array}{l}\mathrm{ER} \\
R E\end{array}$} \\
\hline & 30 & 45 & 60 & 75 & 90 & \\
\hline Número de refeições (nㅇdia) & 13,00 & 18,25 & 14,75 & 14,75 & 13,25 & $\hat{\mathrm{Y}}=14,80$ \\
\hline Number of meals (n./day) & & & & & & \\
\hline $\begin{array}{l}\text { Tempo por refeição (min) } \\
\text { Meal time }\end{array}$ & 22,85 & 13,24 & 13,43 & 9,82 & 8,80 & 1 \\
\hline
\end{tabular}

1. $\hat{\mathrm{Y}} 26,2358-0,2101^{\star *} \mathrm{C}, \mathrm{r}^{2}=0,81 ;{ }^{* *}(\mathrm{P}<0,01)$ pelo teste $\mathrm{F}$ (by the $\mathrm{F}$ test) $; \mathrm{e} \mathrm{C}=$ Nível de concentrado nas dietas (Concentrate level in the diets).

Tabela 5 - Médias da freqüência do número de refeições diárias, em relação ao tempo despendido por refeição, em função dos níveis de concentrado das dietas

Table 5 - Means of the frequency of the daily meals number, in relation to the spending meal time, according to the concentrate levels in the diets

Tempo por refeição (min)

Meal time

Níveis de concentrado (\%)

Concentrate levels

\begin{tabular}{lccccc} 
& 30 & 45 & 60 & 75 & 90 \\
\hline $5 \longmapsto 15$ & 3,50 & 11,50 & 8,25 & 12,25 & 11,50 \\
$15 \longmapsto 25$ & 4,50 & 4,75 & 4,25 & 1,75 & 1,75 \\
$25-35$ & 2,00 & 0,50 & 1,50 & 0,75 & 0 \\
$35 \longmapsto 45$ & 1,25 & 0,75 & 0,50 & 0 & 0 \\
$45-55$ & 1,50 & 0,75 & 0,25 & 0 & 0 \\
Mais de 55 & 0,25 & 0 & 0 & 0 & 0 \\
More than 55 & & & & & \\
\hline
\end{tabular}

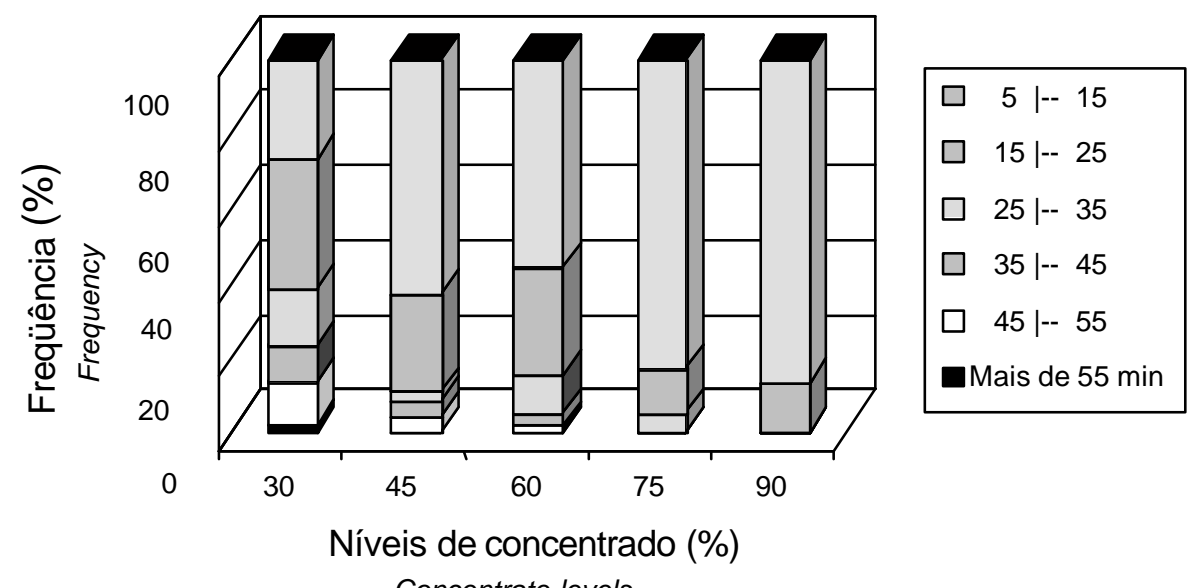

Figura 2 - Freqüência do número de refeições diárias, em relação ao tempo por refeição (min), em função dos níveis de concentrado das dietas.

Figure 2 - Frequency of daily meals numbers, in relation to the meal time (min), according to the concentrate levels in the diets. 


\section{Conclusões}

O tempo despendido em alimentação e ruminação diminuiu e o tempo de ócio aumentou, linearmente, com o aumento do nível de concentrado nas dietas.

A eficiência de ruminação, em $\mathrm{g} M S / \mathrm{h}$, da dieta completa, aumentou linearmente e, quando expressa na fração de volumoso da dieta, diminuiu linearmente com o nível de concentrado.

O aumento do nível de concentrado nas dietas não influenciou o número de refeições diárias, mas diminuiu linearmente o tempo despendido por refeição.

\section{Referências Bibliográficas}

ALBRIGHT, J.L. 1993. Feeding behavior of dairy cattle. J. Dairy Sci., 76(2):485-498.

ALLEN, M.S. 1997. Relationship between fermentation acid production in the rumen and the requirement for physically effective fiber. J. Dairy Sci., 80(7):1447-1462.

BAE, D.H., WELCH, J.G., SMITH, A.M. 1981. Efficiency of mastication in relation to hay by cattle. J. Anim. Sci., 52(6):1371-1375.

BEAUCHEMIN, K.A. 1991. Effects of dietary neutral detergent fiber concentration and alfalfa hay quality on chewing, rumen function, and milk production of dairy cows. J. Dairy Sci., 74(9):3140-3151.

BEAUCHEMIN, K.A. 1992. Effects of digestive and ruminative mastication on digestion of forage by cattle. Anim. Feed. Sci. Techn., 40(1):41-56.

BEAUCHEMIN, K.A., IWAASA, A.D. 1993. Eating and ruminating activities of cattle fed alfalfa or orchard-grass, harvested at two stages of maturity. Can. J. Anim. Sci., 73(1):79-88.

BOSCH, M.W., LAMMERS-WIENHOFEN, S.C.W., BANGMA, G.A. et al. 1992. Influence of stage of maturity of grass silages on digestion processes in dairy cows. 2 . Rumen contents, passage rates, distribution of rumen and faecal particles and mastication activity. Lvstck. Prod. Sci., 32(3):265-281.

BÜRGER, P.J., PEREIRA, J.C., COELHO DA SILVA, J.F. et al. 2000. Consumo e digestibilidades aparentes total e parcial em bezerros holandeses alimentados com dietas contendo diferentes níveis de concentrado. Rev. bras. zootec., 29(1):206-214.

DULPHY, J.P., REMOND, B., THERIEZ, M. 1980. Ingestive behaviour and related activities in ruminants. In: RUCKEBUSH, Y., THIVEND, P. (Eds.). Digestive physiology and metabolism in ruminants. Lancaster: MTP. p.103-122.

FORBES, J.M. 1995. Voluntary food intake and diet selection in farm animals. Wallingford: CAB. 532p.
HAFEZ, E.S.E., BOUISSOU, M.F. 1975. The behaviour of cattle. In: HAFEZ, E.S.E. (Ed.). The behaviour of domestic animals. 3.ed. Baltimore: Williams \& Wilkins. 532p.

JOHNSON, T.R., COMBS, D.K. 1991. Effects of prepartum diet, inert rumen bulk, and dietary polythylene glicol on dry matter intake of lactating dairy cows. J. Dairy Sci., 74(3):933944.

KENNEDY, P.M., McSWEENEY, C.S., FFOULKES, D. et al. 1992. Intake and digestion in swamp buffaloes and cattle. 1 . The digestion of rice straw (Oriza sativa). J. Agric. Sci., 119(2):227-242.

LIVSHIN, N., MALTZ, E., EDAN, Y. 1995. Regularility of dairy cows feeding behavior with computer-controlled feeders. J. Dairy Sci., 78(2):296-304.

MacLEOD, G.K., COLUCCI, P.E., COORE, A.D. et al. 1994. The effects of feeding frequency of concentrates and feeding sequence of hay on eating behaviour, ruminal environment and milk production in dairy cows. Can. J. Anim. Sci., 74(1):103-113.

MOORE, J.A., POORE, M.H., SWINGLE, R.S. 1990. Influence of roughage source on kinetics of digestion and passage, and on calculated extends of ruminal digestion in beef steers fed $65 \%$ concentrate diets. J. Anim. Sci., 68(12):3412-3420.

POLLI, V.A., RESTLE, J., SENNA, D.B. 1995. Comportamento de bovinos e bubalinos em regime de confinamento. I. Atividades. Ciência Rural, 25(1):127-131.

POLLI, V.A., RESTLE, J., SENNA, D.B. et al. 1996. Aspectos relativos à ruminação de bovinos e bubalinos em regime de confinamento. R. Bras. Zootec., 25(5):987-993.

THIAGO, L.R.L., GILL, M., SISSONS, J.W. 1992. Studies of conserving grass herbage and frequency of feeding in cattle. Brit. J. Nutr., 67(3):339-336.

TRAXLER, M.J., FOX, D.G., PERRY, T.C. et al. 1995. Influence of roughage and grain processing in high-concentrate diets on the performance of long-fed steers. J. Anim. Sci., 73(7):1888-1900.

UNIVERSIDADE FEDERAL DE VIÇOSA - UFV. 1997. SAEG - Sistema de análises estatísticas e genéticas. Versão 7.1. Viçosa, MG. 150p. (Manual do usuário).

VAN SOEST, P.J. 1994. Nutritional ecology of the ruminant. 2.ed. Ithaca: Cornell. $476 \mathrm{p}$.

WELCH, J.G. 1982. Rumination, particle size and passage from the rumen. J. Anim. Sci., 54(4):885-894.

WELCH, J.G., HOOPER, A.P. 1988. Ingestion of feed and water. In: CHURCH, D.C. (Ed.). The ruminant animal: digestive physiology and nutrition. Englewood Cliffs:Reston. p.108-116.

Recebido em: 01/03/99

Aceito em: 02/08/99 